\title{
Blood Glucose Level Measurement as an Early Detection to Prevent the Incidence of Feline Diabetes Mellitus in Veterinary Medicine Faculty of Brawijaya University.
}

\author{
Agri Kaltaria Anisa *, Aulanni'am, Dhita Evi Aryani, Wawit \\ Faculty of Veterinary Medicine, Brawijaya University*email: agri_kaltariaa@ub.ac.id
}

\begin{abstract}
Diabetes Mellitus (DM) is a chronic condition of carbohydrate metabolism disorders caused by relative or absolute insulin deficiency. DM is a common disease not only in humans, but also pet animals such as cats. The most common type of diabetes in cats, also known as Feline Diabetes Mellitus (FDM), is type 2 diabetes mellitus with a prevalence of 1:100-1:500. The incidence of FDM can be prevented by an effort of early detection through blood glucose measurement. A cat is diagnosed with FDM if there is a persistent conditions of hyperglycemia with blood glucose levels $>220 \mathrm{mg} / \mathrm{dL}$. This study was aimed to determine the blood glucose level profile of cat patients at the Educational Animal Clinic of Veterinary Medicine Faculty, Brawijaya University as an early detection to prevention FDM in cat patients. The samples used are inpatient and outpatient cats that meet the criteria of "time limit", which is between the periods of 1 to 31 July 2016. This study used a prospective descriptive analysis. The results showed that out of 47 cat patients that were measured its blood glucose level, 39 cats $(82.98 \%)$ had blood glucose levels below normal $(<90 \mathrm{mg} / \mathrm{dL}), 6$ cats $(12.78 \%)$ had normal blood glucose levels $(90-120 \mathrm{mg} / \mathrm{dL})$ and 2 cats $(4.25 \%)$ had blood glucose levels above normal (>120 mg/dL) but still below $220 \mathrm{mg} / \mathrm{dL}$. By comparing the data of blood glucose levels it can be concluded that two of the cat patient that received health care at the Educational Animal Clinic of Veterinary Medicine Faculty, Brawijaya University, during this study, had the potential to suffer from Feline Diabetes Mellitus.
\end{abstract}

Keywords: Feline diabetes mellitus, Blood glucose, Hyperglycemia

\section{Introduction}

Diabetes mellitus (DM) is a common disease in cats with a prevalence of 1:100-1: 500. The most common type of diabetes in cats is type 2 diabetes. Blood glucose measurement is commonly performed as a laboratory analysis to confirm the presence of the Diabetes Mellitus in cats. A cat can be diagnosed with diabetes mellitus if there are persistent conditions of hyperglycemia with blood glucose levels> $220 \mathrm{mg} / \mathrm{dL}$. One of the major risk factors for diabetes mellitus in cats is obesity. Obesity can be caused by feeding (diet) that contains too many carbohydrates. Carbohydrates are not good for cats because it can damage the pancreas. Pancreas damage may lead to the development of insulin resistance and diabetes mellitus [1,2,3].

Lack of public knowledge (cat owners) about Feline Diabetes Mellitus (FDM) in cats becomes its own problems, along with the increasing frequency of occurrence of the disease. The high cost of treatment of diabetes with insulin is also an obstacle for pet owners. Therefore it is necessary to do an early detection of FDM, one way is to perform blood glucose levels measurement, as early prevention of FDM in cat.

\section{Objective}


The objective of this study was to determine the blood glucose profile from the results of blood glucose levels measurement in cat patients (in patients and out patients care) at Veterinary Medicine Faculty of Brawijaya University's Education Animal Clinic. This was done as one of the early steps to prevent the incidence of Feline Diabetes Mellitus (FDM), especially in patients and out patients care at Veterinary Medicine Faculty of Brawijaya University's Education Animal Clinic.

\section{Method}

This study was conducted as an observational study, where the researchers remotely measured the blood sugar levels of cat patients (in patients and out patients) at the Educational Animal Clinic of Veterinary Medicine Faculty, Brawijaya University. The populations used in this study were all patients' cats' inpatient and outpatient care at the Educational Animal Clinic of Veterinary Medicine Faculty, Brawijaya University. The sample used were cat patients(inpatient and outpatient care) that meets the criteria of a time limit ("time limit"), which is between the period 1 to 31 July 2016. This study used a prospective descriptive analysis.

\section{Result and Discussion}

The results of the study, that was done during the period 1 to 31 July 2016 at the Educational Animal Clinic of Veterinary Medicine Faculty, Brawijaya University, prospectively obtained a sample of 47 cat patients (inpatients and outpatients care), which consisted of 20 male cats and 27 female cats. The age range of the sample taken was between 3 months to 5 years old, with the majority of the cats are from domestic breed. Blood glucose level were categorized into four groups, namely hypoglycemia (blood glucose <90 mg / dL), normal (90 mg / dL - $120 \mathrm{mg} / \mathrm{dL}$, hyperglycemia (121 mg / dL - $219 \mathrm{mg} / \mathrm{dL}$ ) and diabetes mellitus ( $\geq 220 \mathrm{mg} / \mathrm{dL}$ ).

The measurement of the blood sugar levels of 47 cat patients, obtained a total of 39 cats $(82.98 \%)$ had blood glucose levels below normal (hypoglycemia) i.e. <90mg/dL, 6 cats (12.78\%) had normal blood glucose levels between $90-120 \mathrm{mg} / \mathrm{dL}$ and 2 cats $(4.25 \%)$ had blood glucose levels above normal (hyperglycemia) i.e. 121-219 $\mathrm{mg} / \mathrm{dL}$, but none of the measured cats had a blood glucose $\geq 220 \mathrm{such} / \mathrm{dLasshown}$ in table 1 and figure 1. Blood glucose level has long been considered as a diagnostic tool for diabetes mellitus. It is commonly performed as a laboratory analysis to confirm the presence of the diabetes mellitus in cats. A cat can be diagnosed with diabetes mellitus if there are persistent conditions of hyperglycemia with blood glucose levels $>220 \mathrm{mg} / \mathrm{dL}$. In this study none of the cat patients has blood glucose $\geq 220 \mathrm{mg} / \mathrm{dL}$, so none of the cat patients are diagnosed with FDM. Although none of the cats are diagnosed to have FDM, the two cats with hyperglycemia are considered to be in risk if their blood glucose levels and living habits (diet, exercise etc.) are not monitored and controlled properly.

Table 1 Distribution of Blood Glucose Level of Cat Patients

\begin{tabular}{|l|c|c|}
\hline Blood Glucose Level & $\begin{array}{c}\text { Number of cat } \\
\text { patients }\end{array}$ & Percentage (\%) \\
\hline$\leq 09 \mathrm{mg} / \mathrm{dL}$ & 39 & 82,98 \\
$90-120 \mathrm{mg} / \mathrm{dL}$ & 6 & 12,78 \\
$121 \mathrm{mg} / \mathrm{dL}-219 \mathrm{mg} / \mathrm{dL}$ & 2 & 4,25 \\
$\geq 220 \mathrm{mg} / \mathrm{dL}$ & 0 & 0 \\
\hline Total & 47 & 100 \\
\hline
\end{tabular}


The absence of cat patients that have blood glucose level in the range that is considered as diabetes was supported by supporting data, such as gender, age, body weight and breed of the cat patient samples.

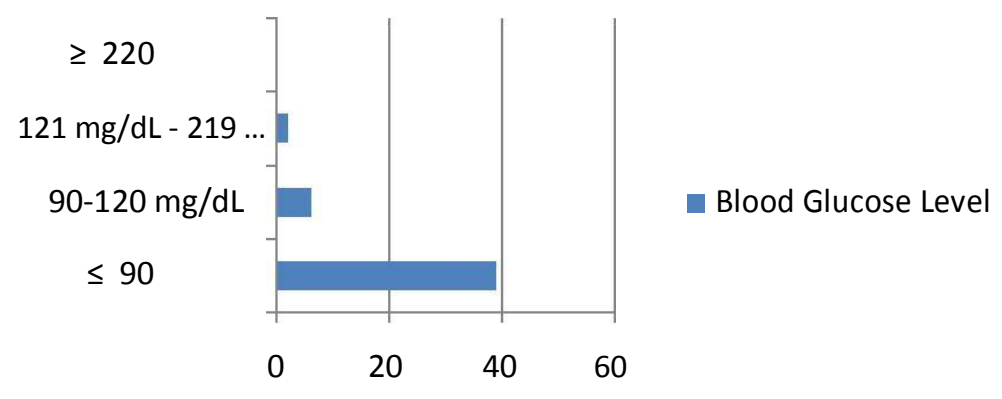

FIGURE 1. Distribution of Blood Glucose Level of the cat patients

Gender, age, obesity and breed are risk factors of diabetes mellitus (DM). Male cats have higher risk to suffer diabetes mellitus compared to female cats. One of the reasons may be that male cats have lower sensitivity to insulin [2]. Meanwhile, the cat patient samples in this study consist of more female cats than male. Related to age, DM/FDM can happen to cats of all ages, but most of the cats that are diagnosed to suffer FDM are those with an age above 6 years old. Meanwhile in this study all of the cat patient samples aged less than 5 years old. Another risk factor of feline diabetes mellitus is obesity. Cats that are obese have a chance of about 4 times more likely to suffer from diabetes compared to cats with optimal weight. That is because obese cats have decreased insulin sensitivity [4].However, the results of this study note that all cat patient samples weight less than $5 \mathrm{~kg}$. In addition to gender, age, and obesity,

a cat's breed is also one of the risk factor was performed in Australia, New Zealand, and England showed that Burmese cats are four

times more at risk to suffer feline diabetes mellitus compared to domestic cat breed [2]. In this study, none of the cats are Burmese breed. Although none of the cat patients in this study are diagnosed to suffer FDM, a routine blood glucose level measurement is recommended to monitor the blood glucose level of the cats to prevent the cats from actually suffering feline diabetes mellitus.

\section{Conclusions}

The conclusion of this study is none of cat patients sampled at Veterinary Medicine Faculty of Brawijaya University's Education Animal Clinic suffers from diabetes mellitus / Feline Diabetes Mellitus, but two cats are considered at risk because they have blood glucose level $121 \mathrm{mg} / \mathrm{dL}-219 \mathrm{mg} / \mathrm{dL}$ (hyperglycemia).

\section{Acknowledgment}

This project supported and fascilitated by Veterinary Medicine Faculty of Brawijaya University, and Biochemistry Department Team.

\section{Reference}

Birchard, S.J. and R.G. Sherding, 2006. Saunder Manual of Small Animal Practice. 3rd Edition. Saunder Elsevier. P.376-389. 